\title{
Application of AHP in the National Scholarship Assessment System for Graduate
}

\author{
De-Bo Fu, Xin Zhang, Fei Cheng
}

The School of Electronics and Information Engineering, Tongji University, Jia Ding, Shang Hai, China

\begin{abstract}
According to the research of the reasonable distribution for the graduate National Scholarship, we set up the hierarchical structure for evaluation index applying with AHP, we can construct judgment matrix and determine the weights with the experience, then we could confirm the graduate National Scholarship assessment. Upon the examination, we could find that the method is fair, impartial, scientific and operable, it provides more scientific reference for National Scholars hip assessment system for graduate.
\end{abstract}

Keywords - National Scholarship for graduate, AHP, Assessment System

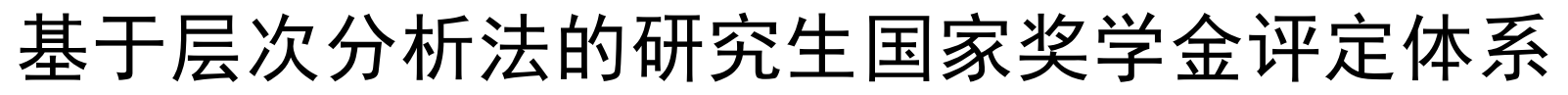

付德波 张金金 程飞

同济大学电子与信息工程学院, 嘉定, 上海, 中国

摘 要 针对研究生国家奖学金评定中的指标确定和合理量化问题, 应用层次分析法, 建立评价指标的递阶层次结构, 结合实践经验构造判断矩阵, 计算指标层对目标层的相对重要性权重, 编制研究生国家奖学金综合评定表。通过实例验证了 所构建评定体系的有效性和合理性, 为科学、公平、公正地评定研究生国家奖学金提供参考。

关键词 研究生国家奖学金，层次分析法，评定体系

\section{1. 引言}

为发展中国特色研究生教育, 促进研究生培养机制改 革，提高研究生培养质量，2012 年 9 月，财政部、教育部 联合制定了《研究生国家奖学金管理暂行办法》, 设立研究 生国家奖学金, 用于奖励普通高等学校中表现优异的全日 制研究生。《办法》指出, 研究生国家奖学金每年奖励 4.5 万名在读研究生, 其中, 博士研究生 1 万名, 硕士研究生 3.5 万名; 博士研究生国家奖学金奖励标准为每生每年 3 万元, 硕士研究生国家奖学金奖励标准为每生每年 2 万元。 《办法》指出, 研究生国家奖学金基本申请条件为: 热爱 社会主义祖国, 拥护中国共产党的领导; 遵守宪法和法律, 遵守高等学校规章制度; 诚实守信, 道德品质优良; 学习 成绩优异, 科研能力显著, 发展潜力突出[1]。该奖学金不 仅有利于激励研究生刻苦学习, 投身科研, 激发潜力, 而 且有利于国家为各行各业培养大量高素质的研究生人才, 从而为“科教强国”战略的实施提供新的有力支撑。经过 2012、2013 两年的实践, 研究生国家奖学金由于奖金额度
大, 奖励人数少, 但是评审指标较为模糊, 多采用定性评 审, 引起普遍关注[2]。

层次分析法[3]、 [4] (Analytical Hierarchy Process, AHP) 由美国著名运筹学家、匹兹堡大学 T.L.Saaty 教授在 20 世 纪 70 年代创立, 是对非定量事件作定量分析的一种有效方 法, 如对施工质量和管理绩效等进行评价[5]、[6], 已广泛 应用于政府决策、企业管理、经济计划、科技管理、干部 选拔、教育规划、资源分配、冲突分析等多个领域 ${ }^{[7]}$ 。本 文针对研究生国家奖学金的特点和学科特点, 以电气工程 学科为例, 建立研究生国家奖学金的指标体系, 并运用层 次分析法确定研究生国家奖学金评定各因素的权重,代替 传统的权重直接取定法, 建立研究生国家奖学金申请人综 合素质评定的多级模型,从而使研究生国家奖学金的评定 更加公正、客观和科学。 


\section{2. 层次分析法理论基础}

\section{1 层次分析法的基本原理}

根据人的思维规律, 面对复杂的选择问题, 将问题分 解成各个组成因素, 再将这些因素按支配关系分组形成递 阶层次结构, 通过两两比较的方式确定层次中各因素的相 对重要性, 然后综合决策者的判断, 确定决策方案相对重 要性的总的排序, 从而作出选择和判断。整个思维过程的 关键是层次的划分、权重的确定和排序的并合规则 ${ }^{[8]}$ 。

\section{2 层次分析法的基本步骤}

（1）构造层次分析模型, 这是层次分析法的关键步骤, 它的好坏直接关系到评价任务完成的质量, 因为其是层次 分析法系统性与综合性的集中表现。根据对问题的了解和 初步分析, 先确定评价的目标, 再明确评价方案的准则, 然后把目标、评价准则连同行动方案一起构造一个层次结 构模型。在这个模型中, 目标、评价准则和行动方案处于 不同的层次, 彼此之间的关系用线段表示。例如最简单的 可以分成三层, 如图 1 所示。

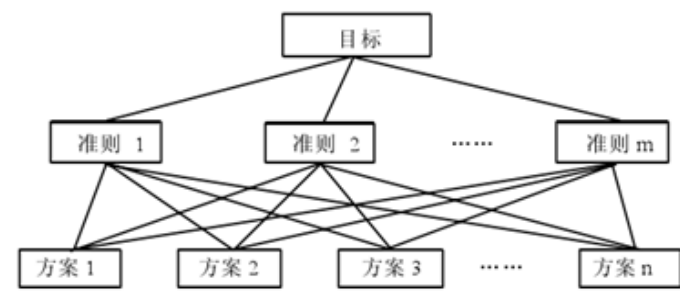

目标层

图 1 层次结构图

(2) 确定权重, 首先要确定分组的指标权重, 然后再 确定层次的指标权重。 权重。

(3) 利用判断矩阵计算被比较元素对于该准则的相对

(4)计算各层元素对系统目标的合成权重,并进行排序。

\section{3 模型假设}

(1) 所有指标准确真实反映了方案层的真实水平;

(2) 各项指标评价权重是客观的;

(3)分配给各项指标的权重只与所计算出的权重有关;

(4) 采用 $1 \sim 9$ 标度法对两个元素 $u_{i}$ 和 $u_{j}$ 的重要性程度 赋值, 记为 $a_{i j}, a_{i j}$ 越大, 则 $u_{i}$ 相对 $u_{j}$ 越重要。

\section{4 相关定义}

定义 1 权重: 在递阶层次结构中, 设上一层元素 $\mathrm{C}$ 为准 则,则其所支配的下一层元素 $\mathrm{u} 1, \mathrm{u} 2 \ldots \mathrm{un}$ 对于准则 $\mathrm{C}$ 相对重
要程度即权重,其中, $n$ 为元素个数。

定义 2 判断矩阵:综合考虑某一层中的各元素,对元素 作两两相对比较, 得到的矩阵为判断矩阵, 表示为

$$
A=\left(a_{i j}\right)_{n \times n}=\left[\begin{array}{cccc}
a_{11} & a_{12} & \ldots & a_{1 n} \\
a_{21} & a_{22} & \ldots & a_{2 n} \\
\ldots & \ldots & \ldots & \ldots \\
a_{n 1} & a_{n 2} & \ldots & a_{n n}
\end{array}\right]
$$

其中, $a_{i j}$ 表示元素 $u_{i}$ 相对于元素 $u_{j}$ 的重要程度,按 $1 \sim$ 9 标度法对其重要性程度赋值。判断矩阵 A 具有下列性质, 即 $a_{i j}>0, a_{i j}=1 / a_{j i}, a_{i i}=1(i, j=1,2 \ldots n)$ 。

定义 3 完全一致性: 若判断矩阵 $\mathrm{A}$ 满足 $a_{i j}=a_{i k} \times a_{k j}(i, j, k=1,2 \ldots n)$,称矩阵 $A$ 具有完全一致性。若矩阵 具有完全一致性, 则表明专家对某一层各元素进行两两比 较时, 其判断保持一致。

定义 4 满意一致性: 当层次总排序随机一致性比例 $\mathrm{CR}=\mathrm{CI} / \mathrm{RI}<0.1$ 时, 即为达到满意一致性。其中, $\mathrm{CI}=(\mathrm{Kmax}-\mathrm{n}) /(\mathrm{n}-1)$, 为层次总排序一致性指标; $\mathrm{RI}$ 为层次 总排序平均随机一致性指标, 其值如表 1 所示; Kmax 为 判断矩阵的最大特征值; $\mathrm{n}$ 为判断矩阵 $\mathrm{A}$ 的阶数。

表 $11 \sim 9$ 阶判断矩阵的平均随机一致性指标

\begin{tabular}{|c|c|c|c|c|c|c|c|c|c|c|}
\hline $\mathrm{n}$ & 1 & 2 & 3 & 4 & 5 & 6 & 7 & 8 & 9 & 10 \\
\hline $\mathrm{RI}$ & 0 & 0 & 0.58 & 0.9 & 1.12 & 1.24 & 1.32 & 1.41 & 1.45 & 1.49 \\
\hline
\end{tabular}

在实际决策中, 专家只能进行估计判断, 不可能保证 给出的判断矩阵具有完全一致性, 满意一致性用于检验专 家判断思维的一致性。

\section{3. 基于层次分析法的研究生国家奖学金评价体系}

用层次分析法作系统分析, 需要把问题层次化, 依据 问题的性质和总目标把问题分解成不同的因素, 根据这些 因素间的相互影响和隶属关系将因素按不同层次聚集组 合, 形成一个多层次的分析结构模型, 并最终将系统分析 归结为最底层相对于最高层的相对重要性权重的确定。

\section{1 建立层次结构模型}

评价因素确定是层次分析法的基础工作之一, 只有根 据评价对象的特点和评价目的选取适当的评价因素，才能 保证评定模型的合理性和科学性。根据研究生国家奖学金 基本申请条件和笔者近两年参与研究生国家评定的实际工 作经验, 并考虑可操作性, 研究生国家奖学金评定的影响 因素应包括社会服务、学习成绩、发表文章、发明专利、 
学科竞赛等 5 项, 每项因素又包含 3-4 项子因素。具体如 图 2 所示。可见影响研究生国家奖学金评定的因素众多, 仅用一个函数不足以表达它们之间错综复杂的关系, 因此 必须将这些因素划分成多个层次, 逐层进行综合评价。

本文将研究生国家奖学金评定模型分为 4 层, 第 1 层 为目标层 $\mathrm{A}$, 即从申请研究生国家奖学金的申请人中, 篮 选出一定数量的合格候选人; 第 2 层为准则层 $\mathrm{B}$, 包括社 会服务、学习成绩、发表文章、发明专利、学科竞赛等, 反映各位申请人的综合素质情况; 第 3 层为指标层 $\mathrm{C}$, 共 有 17 个具有代表性的因素分别对应准则层 B 中各因素; 第 4 层为方案层 $\mathrm{S}$, 即所有申请研究生国家奖学金的研究 生。建立递阶层次结构并确定下层元素之间的隶属关系后, 可得如图 2 所示的研究生国家奖学金评定层次结构图。

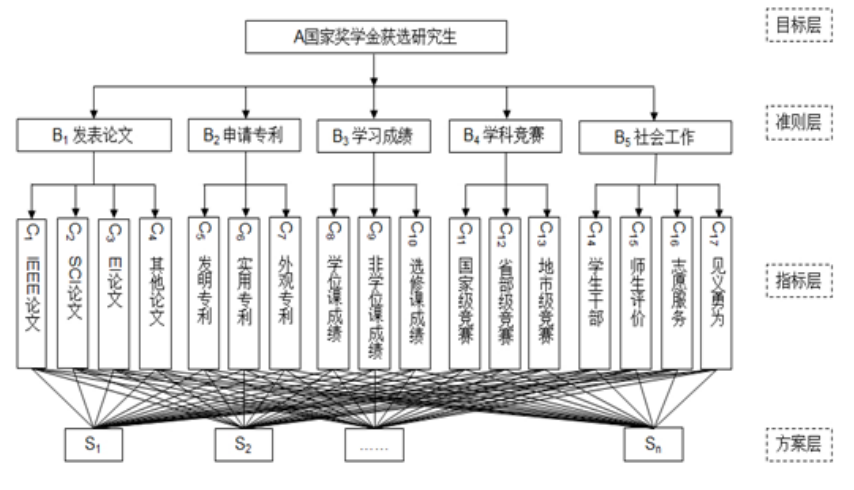

图 2 研究生国家奖学金评定层次结构图

\section{2 构造判断矩阵}

对于研究生国家奖学金评定的因素分析, 要以一定的 分析信息为基础, 这种分析信息的基础主要是人们对于每 一层次上的分组中的两两因素相对重要性所给出的判断。 经过调查, 结合实践经验, 针对判断矩阵的准则, 按 $1 \sim 9$ 标度法赋值, 根据定义 2 , 可得目标层 $\mathrm{A}$ 到准则层 $\mathrm{B}$ 的判 断矩阵、准则层 $\mathrm{B}_{1} 、 \mathrm{~B}_{2} 、 \mathrm{~B}_{3} 、 \mathrm{~B}_{4} 、 \mathrm{~B}_{5}$ 到 $\mathrm{C}$ 的判断矩阵, 如表 2、表 3 所示, 其余省略。

表 2 目标层 $\mathrm{A}$ 到准则层 B 的判断矩阵 A-B

\begin{tabular}{|c|c|c|c|c|c|}
\hline $\mathrm{A}-\mathrm{B}$ & $\mathrm{B}_{1}$ & $\mathrm{~B}_{2}$ & $\mathrm{~B}_{3}$ & $\mathrm{~B}_{4}$ & $\mathrm{~B}_{5}$ \\
\hline $\mathrm{B}_{1}$ & 1 & 5 & 3 & 5 & 5 \\
\hline $\mathrm{B}_{2}$ & $1 / 5$ & 1 & $1 / 2$ & 1 & 1 \\
\hline $\mathrm{B}_{3}$ & $1 / 3$ & 2 & 1 & 1 & 1 \\
\hline $\mathrm{B}_{4}$ & $1 / 5$ & 1 & $1 / 2$ & 1 & 1 \\
\hline $\mathrm{B}_{5}$ & $1 / 5$ & 1 & $1 / 2$ & 1 & 1 \\
\hline
\end{tabular}

表 3 准则层 $\mathrm{B}$ 到 $\mathrm{C}$ 的判断矩阵 B1-C

\begin{tabular}{|c|c|c|c|c|}
\hline $\mathrm{B}_{1}-\mathrm{C}$ & $\mathrm{C}_{1}$ & $\mathrm{C}_{2}$ & $\mathrm{C}_{3}$ & $\mathrm{C}_{4}$ \\
\hline $\mathrm{C}_{1}$ & 1 & 2 & 3 & 4 \\
\hline $\mathrm{C}_{2}$ & $1 / 2$ & 1 & 2 & 2 \\
\hline $\mathrm{C}_{3}$ & $1 / 3$ & $1 / 2$ & 1 & 2 \\
\hline $\mathrm{C}_{4}$ & $1 / 4$ & $1 / 2$ & $1 / 2$ & 1 \\
\hline
\end{tabular}

\section{3 一致性检验}

利用 Matlab 软件, 计算出判断各矩阵的最大特征值, 得到各影响因素的权重, 如表 4 所示。其中, $C R<0.1$, 表明 各判断矩阵具有满意一致性。

表 4 各判断矩阵计算结果

\begin{tabular}{|c|c|c|c|c|c|c|}
\hline 评价指标 & $\begin{array}{l}\text { 矩阵 } \\
\text { A-B }\end{array}$ & $\begin{array}{l}\text { 矩阵 } \\
\mathrm{B}_{1}-\mathrm{C}\end{array}$ & $\begin{array}{l}\text { 矩阵 } \\
\mathrm{B}_{2}-\mathrm{C}\end{array}$ & $\begin{array}{l}\text { 矩阵 } \\
\mathrm{B}_{3}-\mathrm{C}\end{array}$ & $\begin{array}{l}\text { 矩阵 } \\
\mathrm{B}_{4}-\mathrm{C}\end{array}$ & $\begin{array}{l}\text { 矩阵 } \\
\mathrm{B}_{5}-\mathrm{C}\end{array}$ \\
\hline 权重 & $\begin{array}{l}0.5129 \\
0.0988 \\
0.1907 \\
0.0988 \\
0.0988 \\
\end{array}$ & $\begin{array}{l}0.4717 \\
0.2562 \\
0.1644 \\
0.1078\end{array}$ & $\begin{array}{l}0.6 \\
0.3 \\
0.1\end{array}$ & $\begin{array}{c}0.5584 \\
0.3196 \\
0.122\end{array}$ & $\begin{array}{l}0.6 \\
0.3 \\
0.1\end{array}$ & $\begin{array}{l}0.375 \\
0.375 \\
0.125 \\
0.125\end{array}$ \\
\hline 最大特征值 & 5.004 & 4.0458 & 3 & 3.0183 & 3 & 4 \\
\hline CI & 0.001 & 0.0153 & 0 & 0.009 & 0 & 0 \\
\hline RI & 1.12 & 0.89 & 0.52 & 0.52 & 0.52 & 0.89 \\
\hline $\mathrm{CR}$ & 0.0009 & 0.0172 & 0 & 0.0176 & 0 & 0 \\
\hline
\end{tabular}

\section{4 层次总排序计算}

计算同一层次所有因素对于最高层（总目标）相对重 要性的排序权值, 称为层次总排序, 这一过程是最高层次 到最低层次逐层进行的, 若上一层次 $\mathrm{A}$ 包含 $\mathrm{m}$ 个因素 $\mathrm{A}_{1}$ 、 $A_{2} \ldots \ldots A_{m}$, 其层次总排序的权值分别为 $a_{1} 、 a_{2} \ldots \ldots a_{m}$, 下 一层次 $B$ 包含 $n$ 个因素 $B_{1} 、 B_{2} \ldots \ldots B_{m}$, 它们对于因素 $A_{j}$ 的层次单排序的权值分别为 $b_{1 j} 、 B_{2 j} \ldots \ldots B_{n j}$ (当 $B_{k}$ 与 $A_{j}$ 无关时, 取 $\mathrm{B}_{\mathrm{k}}$ 为 0 ), 这一过程是从高层到低层进行的, 如果 $\mathrm{B}$ 层次某些因素对于元素 $\mathrm{A}_{\mathrm{j}}$ 单排序的一致性指标为 $\mathrm{CI}_{\mathrm{j}}$, 相应地平均随机一致性指标为 $\mathrm{RI}_{\mathrm{j}}$, 则 $\mathrm{B}$ 层次总排序 一致性比率为

$$
C R=\sum_{j=1}^{m} a_{j} C_{j} / \sum_{j=1}^{m} a_{j} R I_{j}
$$


类似地, 当 $\mathrm{CR}<0.1$ 时, 认为判断矩阵具有满意的一 致性, 否则就需要调整判断矩阵的元素取值, 使之具有满 意的一致性。

计算得层次总排序一致性比率

$$
C R=\frac{C I}{R I}=\frac{\sum_{i=1}^{17} a_{i} C I_{i}}{\sum_{i=1}^{17} a_{i} R I_{i}}=\frac{0.009}{0.7479}=0.012<0.1
$$

表明层次总排序通过一致性检验, 二级指标权向量可 作为评判研究生国家奖学金获得资格的依据。

根据上述判断矩阵排序的结果, 得到对目标层的优劣 程度的判断, 得到指标层对目标层的优劣程度的判断向量, 进而得到各指标的权重 $\mathrm{C}_{1} \ldots . . \mathrm{C}_{17}$ 依次为 $0.2419,0.1314$, $0.0843,0.0553,0.0593,0.0296,0.0099,0.1065,0.0609$, $0.0233,0.0593,0.0296,0.0099,0.0371,0.0371,0.0126$, 0.0126 。

\section{5 编制研究生国家奖学金综合评定成绩表}

在 C 层次总排序中, 经调整后得到各项指标的权重, 从而编制出研究生国家奖学金综合评定成绩表, 如表 5 所 示。表中, “专家考评分” 是由相应专家小组评定出的百分 制分数, 如发表文章, 可由学科专业委员会对研究生国家 奖学金申请人的发表文章情况进行百分制评分; 如社会服 务, 可由班主任、辅导员、导师、班委等组成的专家小组 对申请人的社会服务情况进行百分制评分。经专家小组对 各位申请人的各项指标进行综合打分, 计算出对应指标层 的权重分, 再对权重分求和, 从而得到各位申请人的综合 评定总分, 依总分的高低排列顺序, 再根据奖学金名额, 即可遴选出适当数量的研究生国家奖学金的候选人。

\section{6 编制研究生国家奖学金综合评定成绩表}

假设某高校电气工程学科 2013 年有 3 名研究生国家奖 学金指标, 共有 6 名研究生申请, 相应专家小组对 6 名研 究生的指标分评定之后, 用《研究生国家奖学金评定表》, 可得出 6 位申请人甲乙丙丁戊己的综合评定分数依次为 $76.9745 ， 34.0055 ， 67.1995 ， 52.025 ， 46.2715 ， 44.7985$ 。 将各位同学综合评定分数由高到低排列, 显然甲丙丁三位 研究生当选研究生国家奖学金候选人, 符合专家小组的预 期, 且能有效说服其他落选的申请人。
表 5 某研究生国家奖学金综合评定表

\begin{tabular}{|c|c|c|c|}
\hline 准则层权重 & 指标层权重 & 专家考评分 $(\mathrm{Pi})$ & 权重分 (Wci*Pi) \\
\hline \multirow{4}{*}{$\begin{array}{c}\text { 发表文章 } \\
\mathrm{WB}_{1}=0.5129\end{array}$} & $\mathrm{WC}_{1}=0.2419$ & $\mathrm{P}_{1}$ & $\mathrm{WC}_{1} * \mathrm{P}_{1}$ \\
\hline & $\mathrm{WC}_{2}=0.1314$ & $\mathrm{P}_{2}$ & $\mathrm{WC}_{2} * \mathrm{P}_{2}$ \\
\hline & $\mathrm{WC}_{3}=0.0843$ & $\mathrm{P}_{3}$ & $\mathrm{WC}_{3} * \mathrm{P}_{3}$ \\
\hline & $\mathrm{WC}_{4}=0.0553$ & $\mathrm{P}_{4}$ & $\mathrm{WC}_{4} * \mathrm{P}_{4}$ \\
\hline \multirow{3}{*}{$\begin{array}{c}\text { 申请专利 } \\
\mathrm{WB}_{2}=0.0998\end{array}$} & $\mathrm{WC}_{5}=0.0593$ & $\mathrm{P}_{5}$ & $\mathrm{WC}_{5} * \mathrm{P}_{5}$ \\
\hline & $\mathrm{WC}_{6}=0.0296$ & $\mathrm{P}_{6}$ & $\mathrm{WC}_{6} * \mathrm{P}_{6}$ \\
\hline & $\mathrm{WC}_{7}=0.0099$ & $\mathrm{P}_{7}$ & $\mathrm{WC}_{7} * \mathrm{P}_{7}$ \\
\hline \multirow{3}{*}{$\begin{array}{c}\text { 学习成绩 } \\
\mathrm{WB}_{3}=0.1907\end{array}$} & $\mathrm{WC}_{8}=0.1065$ & $\mathrm{P}_{8}$ & $\mathrm{WC}_{8} * \mathrm{P}_{8}$ \\
\hline & $\mathrm{WC}_{9}=0.0609$ & $\mathrm{P}_{9}$ & $\mathrm{WC}_{9} * \mathrm{P}_{9}$ \\
\hline & $\mathrm{WC}_{10}=0.0233$ & $\mathrm{P}_{10}$ & $\mathrm{WC}_{10} * \mathrm{P}_{10}$ \\
\hline \multirow{3}{*}{$\begin{array}{c}\text { 学科竞赛 } \\
\mathrm{WB}_{4}=0.0998\end{array}$} & $\mathrm{WC}_{11}=0.0593$ & $\mathrm{P}_{11}$ & $\mathrm{WC}_{11} * \mathrm{P}_{11}$ \\
\hline & $\mathrm{WC}_{12}=0.0296$ & $\mathrm{P}_{12}$ & $\mathrm{WC}_{12} * \mathrm{P}_{12}$ \\
\hline & $\mathrm{WC}_{13}=0.0099$ & $\mathrm{P}_{13}$ & $\mathrm{WC}_{13} * \mathrm{P}_{13}$ \\
\hline \multirow{4}{*}{$\begin{array}{c}\text { 社会服务 } \\
\mathrm{WB}_{5}=0.0994\end{array}$} & $\mathrm{WC}_{14}=0.0371$ & $\mathrm{P}_{14}$ & $\mathrm{WC}_{14} * \mathrm{P}_{14}$ \\
\hline & $\mathrm{WC}_{15}=0.0371$ & $\mathrm{P}_{15}$ & $\mathrm{WC}_{15} * \mathrm{P}_{15}$ \\
\hline & $\mathrm{WC}_{16}=0.0126$ & $\mathrm{P}_{16}$ & $\mathrm{WC}_{16} * \mathrm{P}_{16}$ \\
\hline & $\mathrm{WC}_{17}=0.0126$ & $\mathrm{P}_{17}$ & $\mathrm{WC}_{17} * \mathrm{P}_{17}$ \\
\hline \multicolumn{3}{|c|}{ 测评总分 } & $\mathrm{WC}=\Sigma\left(\mathrm{Wc}_{\mathrm{i}} * \mathrm{P}_{\mathrm{i}}\right)$ \\
\hline
\end{tabular}

\section{4. 结语}

本文针对研究生国家奖学金分配过程中影响因素复杂 且难以定量评价的问题, 运用层次分析法, 通过研究生国 家奖学金综合评定专家对申请人的各项指标进行综合打 分, 然后求出这些指标的加权和, 从而得到申请人的综合 测评总分, 依综合测评分数的高低顺序, 篮选出一定数量 的候选人。

层次分析法把对象作为一个系统, 按照分解、比较判 断、综合的思维方式进行决策, 与一般的方法相比, 更具 系统性和实用性。本文所建立的模型克服了研究生国家奖 学金评定的主观随意性, 提高了评定过程和结果的科学性、 公平性和公正性。模型适用于具有相同影响因素的单位内 研究生国家奖学金评定, 如某系、某学科; 由于不同学科、 专业之间影响因素差异较大, 如公共管理学科和电气工程 学科之间, 该模型的权重需要重新确定。模型可以推广运 用到研究生国家奖学金的指标分配上, 比如教育部（省市 
教育厅 (局)) 对各高校的指标分配、高校研究生主管部门 对院系指标的分配, 都可以根据相应影响因素, 构建出合 适的模型, 更加科学、公平、公正的分配研究生国家奖学 金指标。

但是值得注意的是, 应用层次分析法对研究生国家奖 学金申请人进行综合评估, 简洁高效, 但应用过程中必然 涉及到大量矩阵运算及最大特征值求解, 计算量较大, 只 有借助相关的数学软件如 Matlab 软件编程求解才能更加 方便。

\section{参考文献(References)}

[1] Ministry of Education: Notice of "The Interim Measures of National Scholarship for graduate" [EB/OL]. http://www.moe.gov.cn/publicfiles/business/htmlfiles/moe/moe_ 1779/201210/143547.html/2014-02-03.

[2] Wei Zhang. The Accreditation Status and Countermeasures of National Scholarship for graduate. Management observed, 2013,
33: $55-56$.

[3] Lian-Fen Wan, Shu-Bai Xu. The Introduction of AHP. Bei Jing: China Renmin University Press, 1990: 1-25.

[4] Zhuang suo fa.Study of synthetic evaluation model based on Analytical Hierarchy Process. Journal of Hefei University of Technology (Natural Science), 2000, 04: 582-585+590.

[5] Jie Cao,Yi-Wen Yang.The Performance Evaluation for Alliance's Process Management with AHP. Computer Integrated Manufacturing System, 2008, 14(8):1652-1657.

[6] Yi Zhang, Li-Han Zhang. The Asphalt Pavement Construction Quality Assessment Model using AHP. Tongji University (Natural Science), 2011, (39)2:254-258.

[7] Jiang Zou. The Quality Assessment System of College Teachers applying with AHP. Nanchang University (Humanities and Social Sciences), 2007, 06:178-180.

[8] Li-Bo Xiong, Ju-Jiao Xiong. Application of AHP in Comprehensive Quality Evaluation of Graduate. Heilongjiang Education, 2006(5), 90-92. 